\title{
Desarrollo de un sistema de aspas transformable en las cubiertas plegables
}

\section{Development of a transformable blade system on folding roofs}

\author{
Carlos Cesar Morales Guzman $^{(*)}$
}

\section{RESUMEN}

La presente investigación se centra en diseñar y construir un sistema transformable basado en el método desarrollado por el Dr. Félix Escrig, que nos brindó los conceptos para desarrollar una mejor propuesta y generar sistemas transformables más rápidos, por lo cual se construyó una serie de prototipos que verifiquen y validen nuestro proceso de diseño geométrico para crear una estructura plegable.

Por lo que conllevo a analizar los modelos con el software WinTess que realizo la simulación estructural de dichas estructuras plegables con una membrana textil y nos ayudó a verificar la seguridad estructural del modelo, se abordó el diseño del nodo con el software Solid Work que nos auxilió para diseñar los detalles industriales de conexión por medio de CAD (Computer-Aided-Desing) ya que su paquetería tiene la capacidad para desarrollar prototipados experimentales y realizar los detalles estructurales.

Palabras clave: Arquitectura Transformable, Cubiertas Plegables, Simulaciones Constructivas-Estructurales.

\section{ABSTRACT}

The present research focuses on designing and build a transformable system based on the method developed by Dr. Félix Escrig, who gave us the concepts to develop a better proposal and generate transformable systems more rapid, so a series of prototypes were built to verify and validate our geometric design process to create a collapsible structure.

So it was made an analyze the models with the software "WinTess" that realized the structural simulation of the above mentioned of the folded structures with a with a textile membrane and helped us to verify the structural safety of the model, the design of the node was tackled with the software "Solid Work" that helped us to design the industrial connection details by means of CAD (Computer-Aided-Desing) since its package has the capacity to develop experimental prototypes and to make the structural details.

Keywords: Transformable Architecture, Folding Covers, Constructive-Structural Simulations.

(*) Doctor Arquitecto y Candidato a Doctor en Estructuras de la Edificación. Alumno de Doctorado, Escuela Técnica Superior de Arquitectura en Madrid, Universidad Politécnica de Madrid y Profesor-Investigador en la Universidad Veracruzana Poza Ria, Ver, México.

Persona de contacto: dr.arqmorales@gmail.com (Morales Guzman Carlos Cesar)

ORCID: https://orcid.org/oooo-0002-4499-6968 (Morales Guzman Carlos Cesar)

Cómo citar este artículo/Citation: Carlos Cesar Morales Guzman (2021). Desarrollo de un sistema de aspas transformable en las cubiertas plegables. Informes de la Construcción, 73(562): e397. https://doi.org/10.3989/ic.73030

Copyright: (C) 2021 CSIC. Este es un artículo de acceso abierto distribuido bajo los términos de la licencia de uso y distribución Creative Commons Reconocimiento 4.0 Internacional (CC BY 4.0). 


\section{INTRODUCCIÓN}

En la arquitectura actual hay una gran variedad de desarrollos tecnológicos industriales, que aportan una diversidad de sistemas estructurales que pueden concebir un espacio arquitectónico, pero en nuestro caso atenderemos los que necesitan transformase rápidamente.

En el presente la edificación tiende a ser más esbelta y de fácil evolución. Lo que implica el diseño de un sistema estructural más ligero y de rápida manufactura, para conseguir esto, es necesario tener parámetros de diseño que nos ayuden a crear un mapa conceptual, que se usará como guía aproximada para obtener los resultados más acordes para este tipo de estructuras.

Para lograr la concepción de la forma en los sistemas plegables se tomarán tres etapas de desarrollo, primeramente, se abordará la etapa del Diseño Conceptual; el cual se buscará y desarrollara los métodos teóricos de diseño, que ayudara a definir las diferentes tipologías de los sistemas transformables, esto ayudará al siguiente paso que se apoyará en diseñar la geometría de la estructura. Existen métodos para proyectar este tipo de geometrías como; el propuesto por Dr. Félix Escrig y el Arq. Emilio Pérez Piñeiro, etc., los cuales pueden encontrar la forma adecuada, por lo tanto, se hará uso, del método por modelación matemática del Félix Escrig, el cual se tomará para aproximar y adecuar nuestra propuesta y proponer una simplificación al método geométrico en forma descriptiva, que pueda facilitar la construcción modular de un sistema plegable. (1) (2) (3)

La siguiente etapa es el Análisis Estructural; para ello se contempla el uso del software especializado para desarrollar modelos estructurales y modelos geométricos que tomen en cuenta la tipología del diseño conceptual, la simulación de los modelos en este tipo de sistemas estructurales implica definir una aproximación de los detalles constructivos, ya que el detalle de la unión es crucial para la construcción de estos sistemas estructurales. En dicho análisis se precisará la distribución de los esfuerzos basándonos en la reglamentación del LRDF y como actuará sobre el modelo propuesto, en especial en los nudos ya que son articulados, ya que se pretende conseguir una mayor seguridad en la resistencia de la estructura a la hora del desplegado de sus partes y no tener ningún fallo.

El principal logro de esta investigación se basará en el Diseño Final; es por ello por lo que la elaboración de prototipos experimentales ayudara a verificar el manejo de la cubierta plegable y la construcción de la estructura, con el objetivo de que se pueda realizar los detalles constructivos finales para su manufacturación, y así sea más eficiente para monitorear su comportamiento estructural, y compararlos con el uso de otros sistemas.

Para tener una idea de cómo diseñar este tipo de estructuras se considera una recomendación del experto en la materia (figura 1), en la que podemos observar que el Dr. H. Berger, provee una serie de parámetros aproximados para encontrar los pasos del diseño de una estructura ligera, esta tabla servirá como referencia para elaborar el mapa conceptual y elaborar la metodología de investigación en la búsqueda de un sistema plegado tensado. (4) (5)

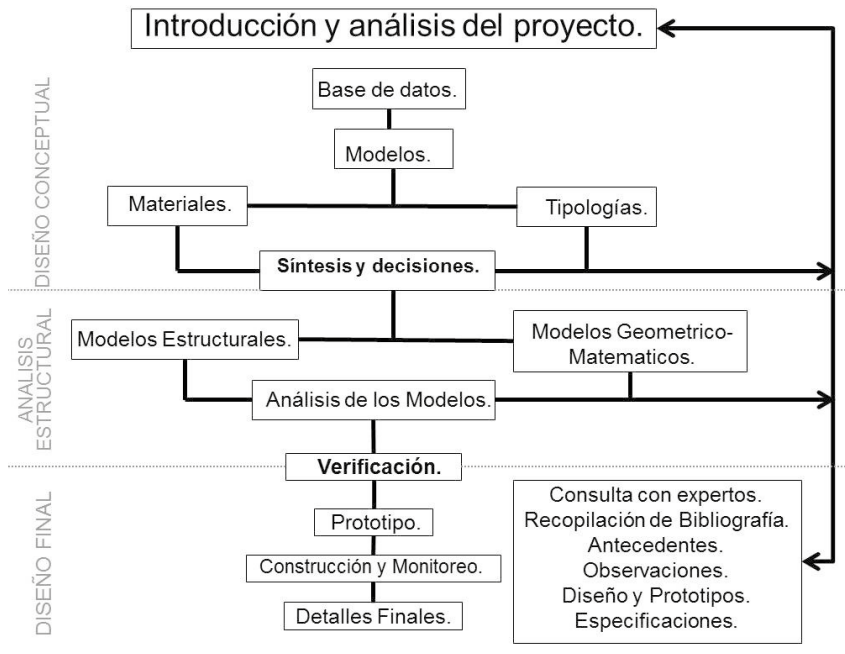

Figura 1. Desarrollo del esquema conceptual del procedimiento de diseño de un sistema plegado tensado, basado en el esquema del Dr. H. Berger, Fuente: Morales (6)

\section{METODO DE DISEÑO}

\subsection{Diseño geometrico conceptual}

Para nuestra investigación la definición de nuestra herramienta será la obtención de la forma de este tipo de estructura, que esta resumida en la siguiente Tabla 1, esta contiene principios basicos que debe poseer una estructura geométricamente plegable. Si esto no está contemplado la flexibilidad estructural del sistema no se dará. Teniendo este principio básico se podrá hacer la traslación matemática más simplificada a una geometría descriptiva que realizara una estructura experimental mucho más rápido y sencilla, para así generarle una serie de iteraciones con los módulos de tijeras, para que la condición de la geometría se dé el razonamiento matemático es simple [1]:

[1] si $\mathbf{a}=\mathbf{a}^{\prime} \mathbf{y} \mathbf{b}=\mathbf{b}^{\prime}$ es una estructura plegable lineal. Que es la misma condición si fuese: $\mathbf{b}+\mathbf{a}^{\prime}=\mathbf{a}+\mathbf{b}^{\prime}$.

Pero si las magnitudes de los valores se inclinaran sobre una de sus variables [2]:

[2] $\mathbf{a}>\mathbf{a}^{\prime} \mathbf{y} \mathbf{b}>$ b' es una estructura plegable curva positiva.

Tabla 1. Formas básicas para generar una estructura plegable, Fuente: Escrig, Morales (11) (6)

\begin{tabular}{|l|l|}
\hline Sistema de Tijera \\
Condición Geométrica Básica
\end{tabular}


Y si se invierte los valores de esta variable [3]:

[3] $\mathbf{a}<$ a' $\mathbf{y} \mathbf{b}<$ b' la curva es negativa.

Esto determinara dicha condición de la geometría, y conllevará posteriormente a generar una serie de propuestas geométricas que comprueben la construcción final de nuestra estructura plegable. (7) (8) (9) (10)

\subsection{Adecuación geométrica experimental.}

La adecuación del modelo experimental anterior se realizó por medio de un método geométrico descriptivo y sus condiciones de diseño se explicaron anteriormente. Para el primer análisis, se utilizará la circunferencia como forma rectora de la figura orgánica de los modelos experimentales, que ayudaran a modular los miembros plegables de cada geometría propuesta en esta adecuación.

Para esto, se dibuja una circunferencia como guía para aplicar dicha traslación (por el momento solo se analizará el ajuste de la forma geométrica), después de colocar el círculo, se trazan dos líneas punteadas que inician del punto "O" de origen y se forma otras dos circunferencias una de mayor diámetro y otra más pequeña, posteriormente se traza un línea como referencia tanto horizontal como vertical en ambas las coordenadas X y Y, estas líneas ayudan a generar las líneas de segmentación del círculo formando puntos de referencia que se utilizan para las divisiones modulares de círculo, en donde se delinearán las figuras geométricas de las aspas formado una gran armadura de ocho secciones.

El proceso genera una figura simple (figuras 2 y 3), para este ejercicio esta sección se copia cinco veces, con lo que determinamos la forma de una cúpula de bóveda corrida, estas se unen por medio de líneas que simulan los arrostramientos y ligaduras, creando las subestructuras geométricas que establecen mayor equilibrio geométrico. (12) (13) (14) (15)
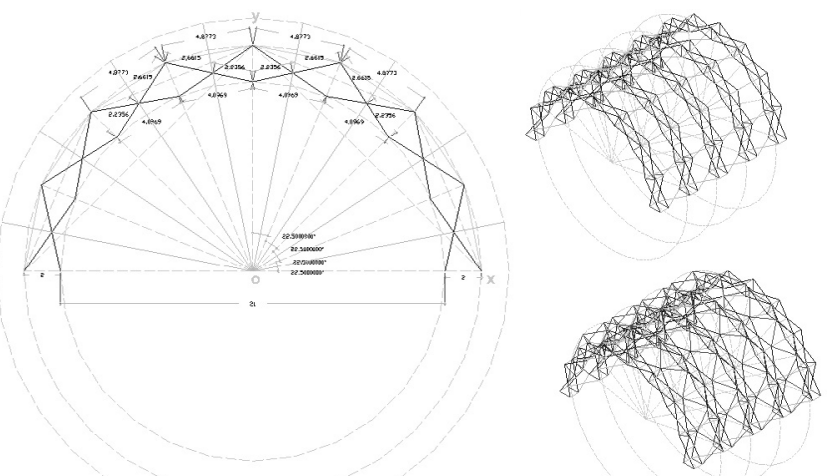

Figura. 2 y 3. Desarrollo de una adecuación orgánica a la geometría, generada por formas y miembros modulares para su estandarización e iteración. Fuente: Morales (9)

Después, se toma la geometría de la armadura circular y se ejecuta la geometría de una cúpula rebajada, esta se forma por medio de dos curvas circulares circunscritas en el centro de la armadura. Siguiendo con el proceso, dichas curvas se copian en su mismo eje hasta formar guías (figura 4, 5), de donde se forman líneas que ayudan a integrar puntos de referencia para dibujar los arrostramientos de la cúpula. Este modelo se adaptará en virtud de reforzar la geometría ya realizada y darle un acabado más estético. (16)
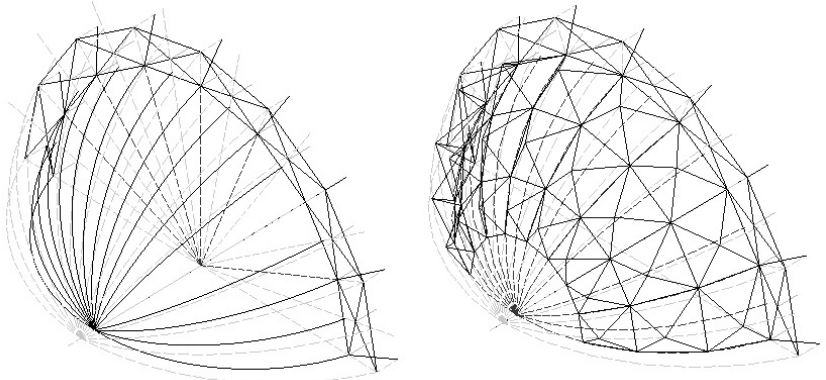

Figuras 4, 5. La adecuación tiene varias formas orgánicas en la que se deben utilizar varias geometrías para crear una estructura flexible. Fuente: Morales (10)

Continuando con el ejercicio para entender cómo se puede desarrollar dicha geometría descriptiva, se desarrolló modelos de adecuación geométrica en varias propuestas, su estructuración y posteriormente el análisis en el espacio (figura 6 , 7), esto brindará los diferentes casos geométricos de unión y de iteración del mismo modelo, ya que en este caso se pueden lograr diferentes procesos geométricos de la estructura, la cual se compondrá de una guía circular que ayudará a formar la figura final. La primera figura está compuesta de dos cuerpos que se comportan como una armadura. (13) (17)
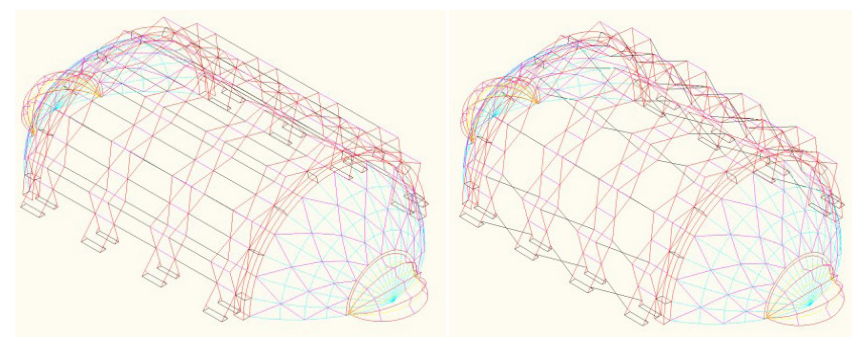

Figura 6, 7. La geometría del modelo se basa en la figura de "X" que forma un armazón que estabiliza a la geometría por forma, está compuesta por dos cubiertas que se unen y componen la figura plegable. Fuente: Morales (10)

La parte más grande del cuerpo geométrico se integra por cuatro armaduras, ligadas líneas rectas que se pliegan en un solo sentido, su figura se compone de miembros de aspas que unidas generan una armadura ayudando a absorber futuros desplazamientos. Las cúpulas de los costados se ajustan con la misma figura de aspas, pero está colocada ya desplegado la cúpula de cañón corrido (figura 8, 9).
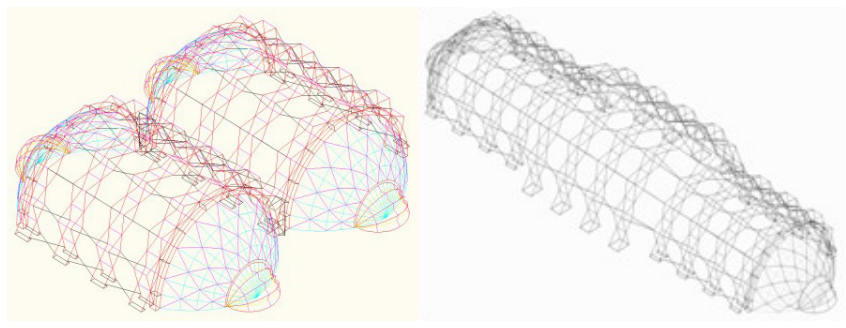

Figuras 8, 9. La geometría puede desarrollarse en diferentes escalas y uniones de diferentes combinaciones, al igual que puede generarse de diferentes piezas en "X" lo cual la hace más estable. Fuente: Morales (10) 


\subsection{Calculo de una estructura transformable.}

La simulación estructura se realiza por medio del software WinTess, desarrollado por el Dr. Sastre de la Universidad Politécnica de Catalunya, el cual desarrolla operaciones de análisis de Segundo orden, por lo que podemos analizar una geometría plegable en su forma estática y con la colocación de una membrana textil, los puntos intermedios de las aspas estarán articuladas al igual que sus extremos por lo que el punto de apoyo de la base de la geometría serán las fijas, dicho lo anterior el análisis estructural del proyecto de la investigación se basa en el rango de factor de seguridad por resistencia y desplazamiento, el código de referencia será con base en el LRFD, que adopta un rango de seguridad para los miembros estructurales de 1 a 1.05 de resistencia del material (acero). Por lo que se considera los siguientes requerimientos de rendimiento para materiales isotrópicos; coeficiente de número de poisson para el acero: .27 para diseñar, para más rigidez .30. Para determinar las cargas se basará por las normas de construcción del Distrito Federal en México, por lo que se selecciona las cargas recomendadas en dicha norma para cubiertas no transitables, dichos parámetros de carga son los siguientes; Carga Muerta: $30 \mathrm{~kg} / \mathrm{m} 2$, Carga Viva Máxima: $40 \mathrm{~kg} /$ m2, Carga Viva Accidental: $20 \mathrm{~kg} / \mathrm{m} 2$, en la tabla 2 se desglosa la combinación de cargas que tendrá en la simulación estructural de la estructura, que afecta solo a las cubiertas no transitables. (18) (19) (20)

Tabla 2. Combinación de Cargas por el Reglamento de Construcción del D.F. Fuente: Morales (6) (15)

\begin{tabular}{|c|c|}
\hline $\begin{array}{l}\text { Primera } \\
\text { Combinación: }\end{array}$ & $(\mathrm{CM}(1.4)+\mathrm{CVM}(1.7)$ \\
\hline $\begin{array}{l}\text { Segunda } \\
\text { Combinación: }\end{array}$ & $(\mathrm{CM}(1.1)+\operatorname{CVA}(1.1)+\operatorname{SISX}(1.1)+\operatorname{SISX}(.33)$ \\
\hline $\begin{array}{l}\text { Tercera } \\
\text { Combinación: }\end{array}$ & $(\mathrm{CM}(1.1)+\operatorname{CVA}(1.1)+\operatorname{SISX}(1.1)-\operatorname{SISX}(.33)$ \\
\hline $\begin{array}{l}\text { Cuarta } \\
\text { Combinación: }\end{array}$ & $(\mathrm{CM}(1.1)+\operatorname{CVA}(1.1)-\operatorname{SISX}(1.1)+\operatorname{SISX}(.33)$ \\
\hline $\begin{array}{l}\text { Quinta } \\
\text { Combinación: }\end{array}$ & $(\mathrm{CM}(1.1)+\operatorname{CVA}(1.1)-\operatorname{SISX}(1.1)-\operatorname{SISX}(.33)$ \\
\hline $\begin{array}{l}\text { Sexta } \\
\text { Combinación: }\end{array}$ & $(\mathrm{CM}(1.1)+\mathrm{CVA}(1.1)+\operatorname{SISZ}(.33)+\operatorname{SISZ}(1.1)$ \\
\hline $\begin{array}{l}\text { Séptima } \\
\text { Combinación: }\end{array}$ & $(\mathrm{CM}(1.1)+\mathrm{CVA}(1.1)+\operatorname{SISZ}(.33)-\operatorname{SISZ}(1.1)$ \\
\hline $\begin{array}{l}\text { Octava } \\
\text { Combinación: }\end{array}$ & $(\mathrm{CM}(1.1)+\mathrm{CVA}(1.1)-\operatorname{SISZ}(.33)+\operatorname{SISZ}(1.1)$ \\
\hline $\begin{array}{l}\text { Novena } \\
\text { Combinación: }\end{array}$ & $(\mathrm{CM}(1.1)+\mathrm{CVA}(1.1)-\operatorname{SISZ}(.33)-\operatorname{SISZ}(1.1)$ \\
\hline $\begin{array}{l}\text { Decima } \\
\text { Combinación: }\end{array}$ & $(\mathrm{CM}(1.1)+\mathrm{CVA}(1.1)+\mathrm{WX}(1.1)$ \\
\hline $\begin{array}{l}\text { Onceava } \\
\text { Combinación: }\end{array}$ & (CM (1.1) + CVA (1.1) - WX (1.1) \\
\hline $\begin{array}{l}\text { Doceava } \\
\text { Combinación: }\end{array}$ & $(\mathrm{CM}(1.1)+\mathrm{CVA}(1.1)+\mathrm{WZ}(1.1)$ \\
\hline $\begin{array}{l}\text { Treceava } \\
\text { Combinación: }\end{array}$ & $(\mathrm{CM}(1.1)+\mathrm{CVA}(1.1)-\mathrm{WZ}(1.1)$ \\
\hline
\end{tabular}

Así con una relación de esbeltez se determinará [4]:

[4] KL/r de 240 en elementos principales y de 300 en miembros secundarios y de arrostramientos.
De igual manera la combinación de carga para el sistema se manejará por el mismo código de resistencia y estabilidad. También se considera el desplazamiento horizontal y vertical para que no exceda el límite de servicio de la estructura establecido en el reglamento del Distrito Federal. Adicionalmente, se verán todos los gráficos de tensión para verificar en dónde hay mayores esfuerzos y cómo estos interactúan dentro de la estructura. Posteriormente, con esta información se desarrollarán las uniones adecuadas para resistir estos esfuerzos en sus nodos.

Para determinar cómo se resolverá la estabilidad de la estructura, se plantea un ejemplo de traslación geométrica y se analiza la geometría establecida, por medio de la simulación estática, para generar los primeros vestigios de tecnología estructural. Para este ejemplo, se accede a la última adecuación geométrica de los ejercicios anteriores, esta geometría es la menos compleja de realizar en este análisis, pues el sistema está constituido por una serie de marcos plegados en forma de aspas, gracias a esta figura geométrica se equilibra y sustenta debido a su misma forma.

Para esta simulación, se especifica que el material sería un acero A36 con un módulo de elasticidad de $2530 \mathrm{~kg} / \mathrm{cm} 2$ y un factor de Poisson's de 0.3 con densidad de $7.83847 \mathrm{Mton} / \mathrm{m}$, las piezas seleccionadas son de tubos OCXE150 para miembros de unión principal, los arrostriamientos están conformados por la misma denominación, al igual que para las armaduras plegables en el espacio grande, donde sé propusieron las misma piezas tubulares; para no complicar tanto el análisis. (2) (3) (11) (21)

El resultado es refleja el comportamiento de los esfuerzos en la estructura; en la que la simulación estática, brinda una visión más clara de la dimensión que pueden llegar a conformar estas adecuaciones geométricas y cuáles serán sus límites de claro a cubrir. Cabe hacer mención que se consideraron las cargas propuesta por el reglamento y el material escogido para dicho desarrollo experimental. La investigación no destina ninguna función espacial a la estructura, ya que el modelo no cuenta con ninguna funcionalidad en específico. Estos parámetros se toman en cuenta dependiendo de la localización del proyecto, en este caso, se utilizará la localización en Poza Rica, Ver, con una función espacial hipotética de nave industrial. (15)

Esta etapa es esencial para el diseño del sistema la colocación de la membrana. Hay varios métodos para hacerlo, en este caso será por el método virtual, donde se obtendrá la forma. Para ello se apoya con el uso de un software institucional elaborado por el Dr. Ramón Sastre Sastres, profesor de la Escuela Técnica Superior de Arquitectura en la Universidad Politécnica de Catalunya.

Dicho software se llama WinTess versión 3.11 realiza el método virtual por densidades de fuerza. Este método genera una relajación en la membrana para obtener la forma deseada de la cubierta textil (figuras 10,11), en la que primeramente se genera una malla rectangular de $35 \mathrm{~m}$ de ancho que sería la longitud que tendría si fuese una cubierta rígida curva y $27 \mathrm{~m}$ de largo que tiene la cubierta en su dirección lineal. (10) (16) (22)

Se elabora una malla de $27 \mathrm{~m}$ ancho y $9.5 \mathrm{~m}$ de ancho con la cual se generan los puntos de anclaje que se conectaran a la armadura, ya realizada la malla se genera el cálculo para la obtención de la forma por densidad de fuerza y relajación, obteniendo la forma que tendrá esta cuando sea pretensada. 
Se selecciona de la combinación de cargas anteriores la que más afecta a la estructura y se aplica, en WinTess no se hacen operaciones de iteración por combinaciones de carga, pero posteriormente se pueden combinar los resultados y ver qué tipo de cargas son las que más afectan al sistema estructural, en consecuencia, quedaría así las cargas propuestas para dicho programa (tabla 3):

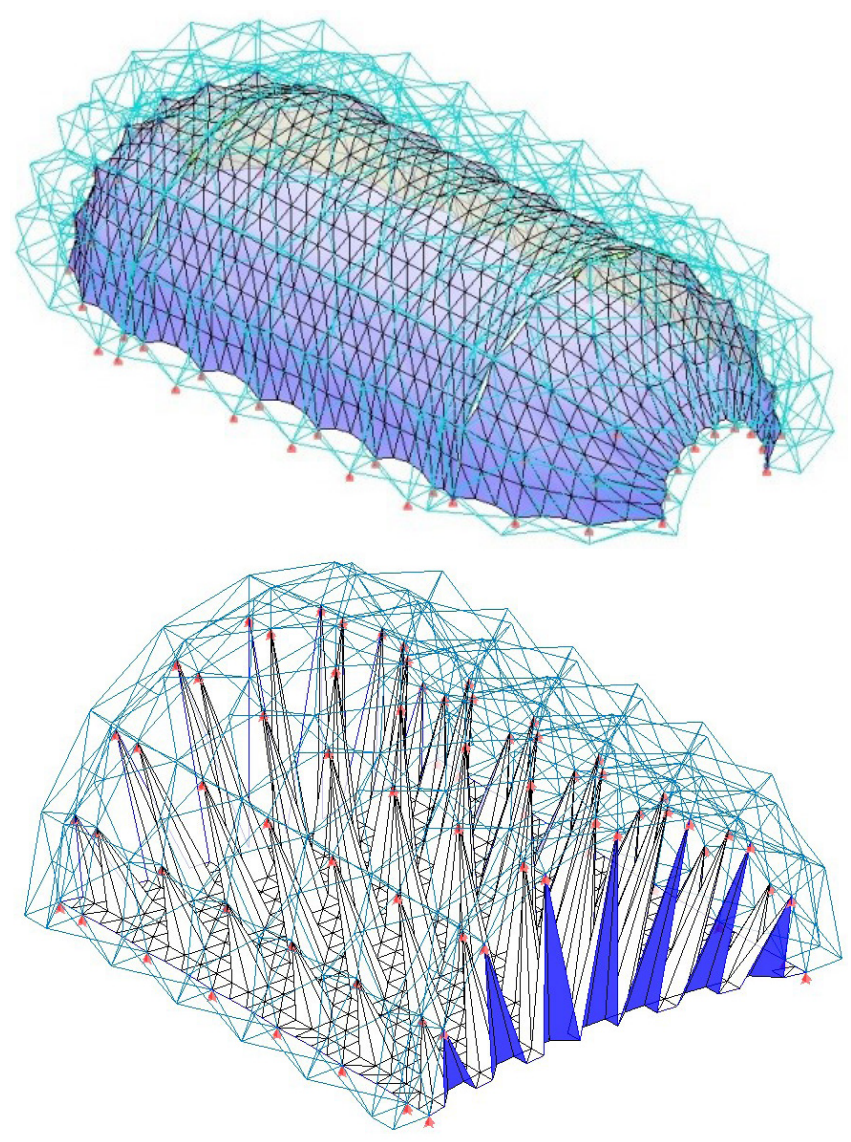

Figuras 10, 11. La búsqueda de la forma también llamado en inglés Form Finding, es parte importante para la investigación, ya que el programa proporciono la rapidez necesaria para terminar las dimensiones exactas de la membrana. Fuente: Morales (6)

Tabla 3. Combinación más afectada para calcularse en el programa WinTess. Fuente: Morales (6) (15)

\begin{tabular}{|c|c|c|}
\hline \multicolumn{3}{|c|}{ WinTess } \\
\hline \multicolumn{3}{|c|}{$\begin{array}{c}\text { Combinación de Carga : }: 1.1+1.1+\text { SISY + 1.1 VIEN X + } \\
.33 \text { VIEN Y }\end{array}$} \\
\hline \multicolumn{3}{|c|}{ Sobrecarga $=20 \mathrm{~kg} / \mathrm{m}^{2}+$ Carga Accidental: $40 \mathrm{~kg} / \mathrm{m}^{2}$} \\
\hline $\begin{array}{l}\text { Viento } X=116.1 \\
\mathrm{~km} / \mathrm{h}\end{array}$ & $\begin{array}{l}\text { Viento } \mathrm{Y}=64.7 \\
\mathrm{~km} / \mathrm{h}\end{array}$ & $\begin{array}{c}\text { Viento total = } \\
132.9109 \mathrm{~km} / \mathrm{h}\end{array}$ \\
\hline \multicolumn{3}{|c|}{ Tipo de edificio $=$ Abierto $(3)>$ Sin $c=0$} \\
\hline \multicolumn{3}{|c|}{ Pretensado de la membrana $=0.08 / 0.08 \%$} \\
\hline \multicolumn{3}{|c|}{ Peso de los cables $=780.8 \mathrm{~kg}$} \\
\hline \multicolumn{3}{|c|}{ Peso de los tubos $=70989.1 \mathrm{~kg}$} \\
\hline \multicolumn{3}{|c|}{ Peso de la membrana $=1464.4 \mathrm{~kg}\left(1394.7 \mathrm{Om}^{2}\right)$} \\
\hline
\end{tabular}

Posteriormente, se realiza el proceso de cálculo por iteración del sistema de aspas, este proceso se realizó con una matriz de nodos por fuerzas desequilibrantes, el programa hace un número de iteraciones hasta lograr que la estructura este equilibrada; el número de iteraciones puede variar, dependiendo de la forma compleja de la estructura. Lo importante de este cálculo, es que se obtiene la dimensión real de los miembros de la estructura, los esfuerzos que actuaran en la misma, las dimensiones de los cables y las deformaciones que pueden tener ya incluida la membrana.

Para este caso, se seleccionó una membrana Serge Ferrari-Fluitop-T2-1002, con una resistencia de $\mathrm{RK}(\mathrm{daN} / 5 \mathrm{~cm})$ 420/420, y unos tubos circulares de $150 \mathrm{~mm}$ de diámetro y 5 $\mathrm{mm}$ de espesor de acero con de denominación europea S235, cables de acero galvanizado de $18 \mathrm{~mm}$ de espesor. Ya realizado el cálculo sobre estas especificaciones, se verifican los datos arrojados del programa, la verificación de los miembros fue realizada por la NCRDF, esto no ocasiona ningún problema, pues los valores que maneja en factores de seguridad son casi iguales que los del manual de acero de LRFD.

En la tabla 4 se observa que todas las barras con mayor esfuerzo de momento y tensión, estos no superan el Ratio de seguridad establecido a la unidad (1), se menciona que, para el tipo de estructura, la comprobación sigue siendo la unidad, pero por comprobación de seguridad es de 1.65 en los tubos, ya que este tipo de estructura no es transitable. (13) (14) (15) (23)

Tabla 4. Se observa que los esfuerzos a considerar siguen siendo los axiles, ya que el sistema funciona como celosía en toda la cubierta. Fuente: Morales (6) (15)

\begin{tabular}{|c|c|c|l|l|}
\hline \multicolumn{5}{|c|}{ TRACCIÓN MAXIMA EN LA MEMBRANA } \\
\hline Barra & Nudos & T/metro & $\mathbf{k g} / \mathbf{5 c m}$ & Ratio \\
\hline 1728 & $487-519$ & 1.470 & 73.5 & 0.90 \\
\hline TRACCIÓN EN LOS CABLES DE RELINGA \\
\hline Cable & T & Ratio & Barra & \\
\hline 1 & 2.860 & 0.75 & $\begin{array}{l}1,2,3,4,5,6,7,8,9,10,11,12,13,14,1 ?- \\
\text { WS-2-(18mm)Galv }\end{array}$ \\
\hline 2 & 2.857 & 0.89 & $\begin{array}{l}45,46,47,48,49,50,51,52,53,54,55,- \\
5 ? W S-2-(18 m m) G a l v\end{array}$ \\
\hline 3 & 1.593 & 0.65 & $\begin{array}{l}65,66,67,68,69,70,71,72,73,74,75,7- \\
? 1 X 91-(18 m m) I n o x\end{array}$ \\
\hline
\end{tabular}

Los datos anteriores generan muchas controversias, ya que no existe un rango exacto de seguridad para este tipo de estructuras pero los especialistas como el Dr. Ramón Sastre Sastre y Dr. José Ignacio Llorens Duran, están redactando reglamentaciones para este tipo de estructuras en Europa y han comprobado que dichas estructuras son factibles con un factor de seguridad de 1,65 es posible para considerar la aleatoriedad en las cargas y en las incertidumbres del modelo de análisis en relación con la geometría real, sin embargo, en este ejercicio, el Ratio se dejó a la unidad por que se manejan factores parciales para cargas y resistencias por la falta de información de este tipo de estructuras, y en nuestro caso, la geometría debe tener rigidez a la hora de estar completamente desplegada y mantenerse en la misma posición para estar en el rango de la seguridad (figura 12, 13). (2) (6) (15) (20)

Los momentos en la cubierta son bajos, ya que la tensión que domina a la estructura es axial, pues su configuración estructural actúa como una viga de celosía, y esto hace que solo actúe los miembros diagonales en su máxima capacidad, y esto hace que funcione mucho mejor el sistema estructural. 

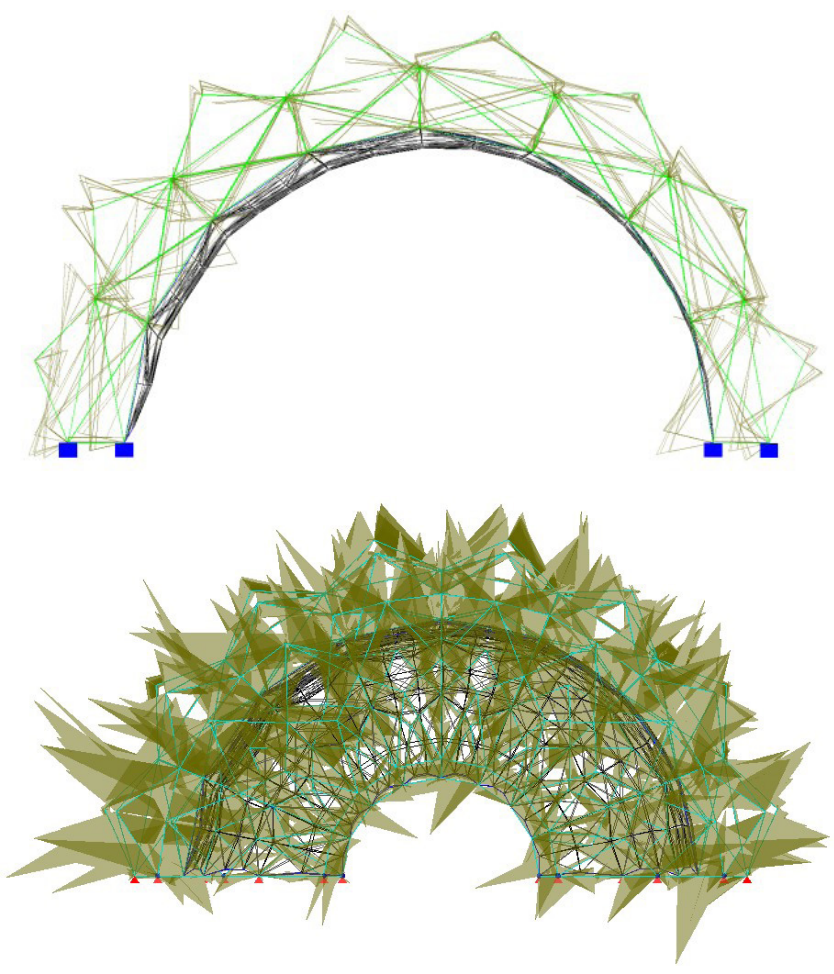

Figuras 12, 13. Los momentos en la cubierta son muy bajos gracias a la disposición de los miembros, ya que la figura de la tijereta funciona como celosía. Fuente: Morales (6)

Siguiendo con los resultados de los análisis del programa podemos verificar también la seguridad estructural del cable y de la membrana textil, este tipo de miembros trabajan solo a tracción, se debe tener cuidado para saber cuánta tensión pueden soportar, por eso la importancia de conocer las propiedades de los materiales y su módulo de elasticidad de estos, ya que determinara la dimensión de la estructura. (tabla 5). (1) (9) (15) (19)

Tabla 5. En la tabla podemos verificar las dimensiones que se deben de contemplar para el diseño constructivo de las conexiones de la membrana y la estructura. Fuente: Morales (15) (24)

\begin{tabular}{|c|c|c|c|c|c|c|c|}
\hline \\
\hline \multicolumn{8}{|c|}{\begin{tabular}{|l|l|l|l} 
Torso & M.max & C.max & Tensión \\
Ratio
\end{tabular}} \\
\hline & $\mathbf{T}$ & Tm & $\mathbf{T m}$ & $\mathbf{T}$ & $\mathrm{kg} / \mathrm{cm}^{2}$ & & \\
\hline 2066 & 15.497 & 0.015 & 0.214 & 0.026 & 1349.6 & 0.95 & Ø150·5_S235 \\
\hline 2462 & 19.036 & 0.002 & 0.252 & 0.123 & 1371.3 & 0.96 & Ø150·5_S235 \\
\hline 2467 & 16.540 & 0.003 & 0.189 & 0.077 & 1373.9 & 0.96 & Ø150:5_S235 \\
\hline 2502 & 16.507 & 0.004 & 0.194 & 0.079 & 1373.5 & 0.96 & Ø150·5_S235 \\
\hline 2512 & 18.782 & 0.005 & 0.216 & 0.105 & 1324.0 & 0.93 & Ø150·5_S235 \\
\hline 2513 & 16.938 & 0.033 & 0.177 & 0.086 & 1223.9 & 0.86 & $\varnothing 150 \cdot 5 \_S 235$ \\
\hline
\end{tabular}

Se propusieron cables de $18 \mathrm{~mm}$, obteniendo valores sobrados, pero para este ejercicio se usarán estos los mismos valores, después de esta simulación se propondrán los detalles constructivos de las conexiones de la cubierta, ya que esas conexiones tendrán contemplada los factores de seguridad del análisis estructural. Por último, se verifica la membrana Serge Ferrari-Fluitop-T2-1002, que resistió perfectamente las deformaciones que tuvo por las presiones de viento, por empuje y succión, con estos referentes, se puede comenzar a diseñar las conexiones reales de la cubierta plegable (figura 14).

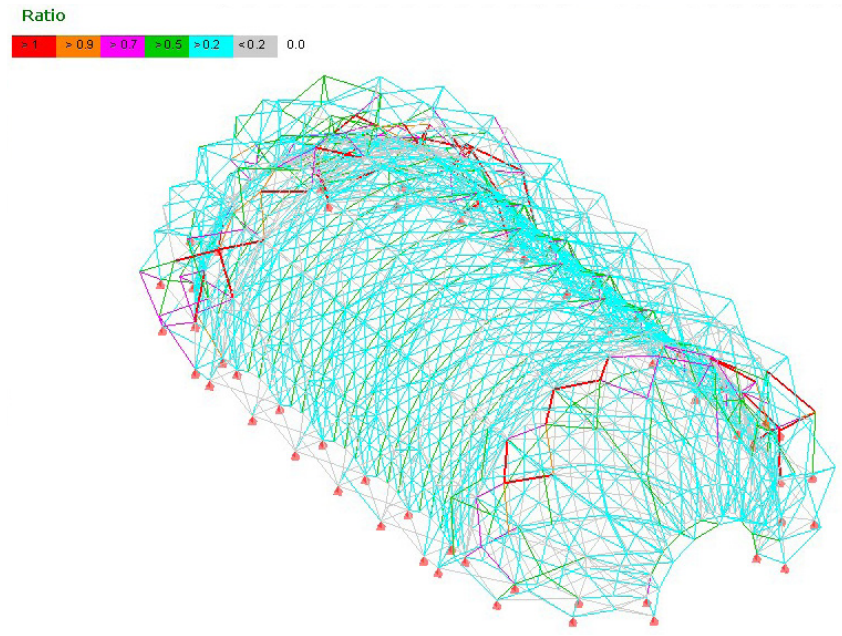

Figura 14. En la figura podemos observar que el Ratio de seguridad que está dentro de los miembros y la membrana de la estructura, no sobrepasa de lo permitido, gracias a ello, se puede dar una propuesta de diseño para las conexiones de la estructura. Fuente: Morales (6) (15)

\subsection{Modelado constructivo de la propuesta final.}

Para adecuar y rectificar la geometría anteriormente descrita se realizará un modelo basándonos en la geometría de referencia que se obtuvo de los modelos aproximados anteriores y así concretar la cubierta final de la siguiente investigación.

Para el desarrollo de la cubierta plegable se realizaría a escala (1:4) con material a base de acero galvanizado. Posteriormente, se realiza la construcción de la cubierta plegable que se describirá en las siguientes figuras donde se proporciona la función y el material con que se realizaron, cada elemento que compone la cubierta transformable tiene la función de estabilizar el sistema plegable de la estructura. El siguiente paso es el armado del modelo, este se desarrolló bajo una serie de conexiones con partes de tubo del mismo acero galvanizado, las uniones articuladas se colocaron con tornillos y tuercas de $5 / 16$, estas resistieron las movilidades del desplegado del modelo y así se pudo construir el prototipo. (figuras 15, 16). (1) (2) (6) (17) (18) (25)

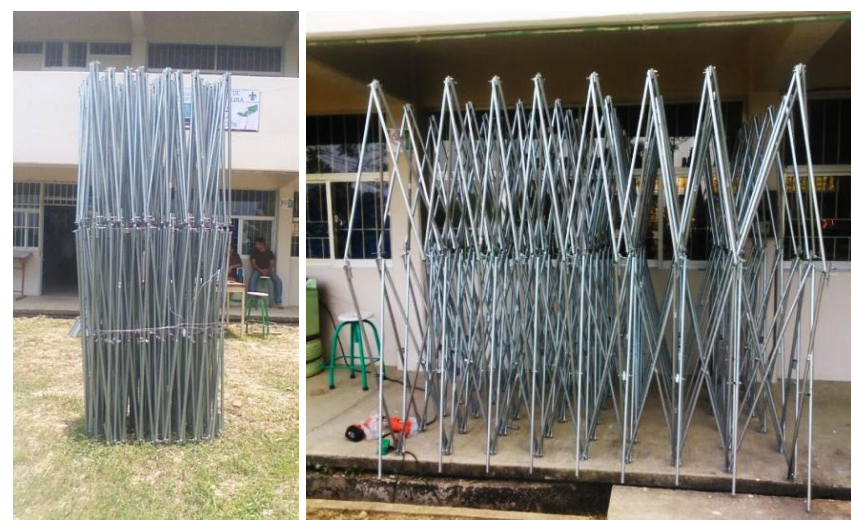

Figuras 15, 16. Una vez construido el paquete de aspas, se realizará el desplegado de la estructura en el sitio para comprobar sus desplegado y plegado de la cubierta transformable. Fuente: Morales (6) (15)

Una vez conectadas las piezas de la cubierta plegable sé sometieron a las pruebas de alzado, con ello se verifican los inconvenientes del modelo, el primer paso es el desplegado 
y el plegado de dicha cubierta, ya que tiene problemas estructurales por los esfuerzos que se llegan a suscitar a la hora del alzado (figuras 17 a la 22).
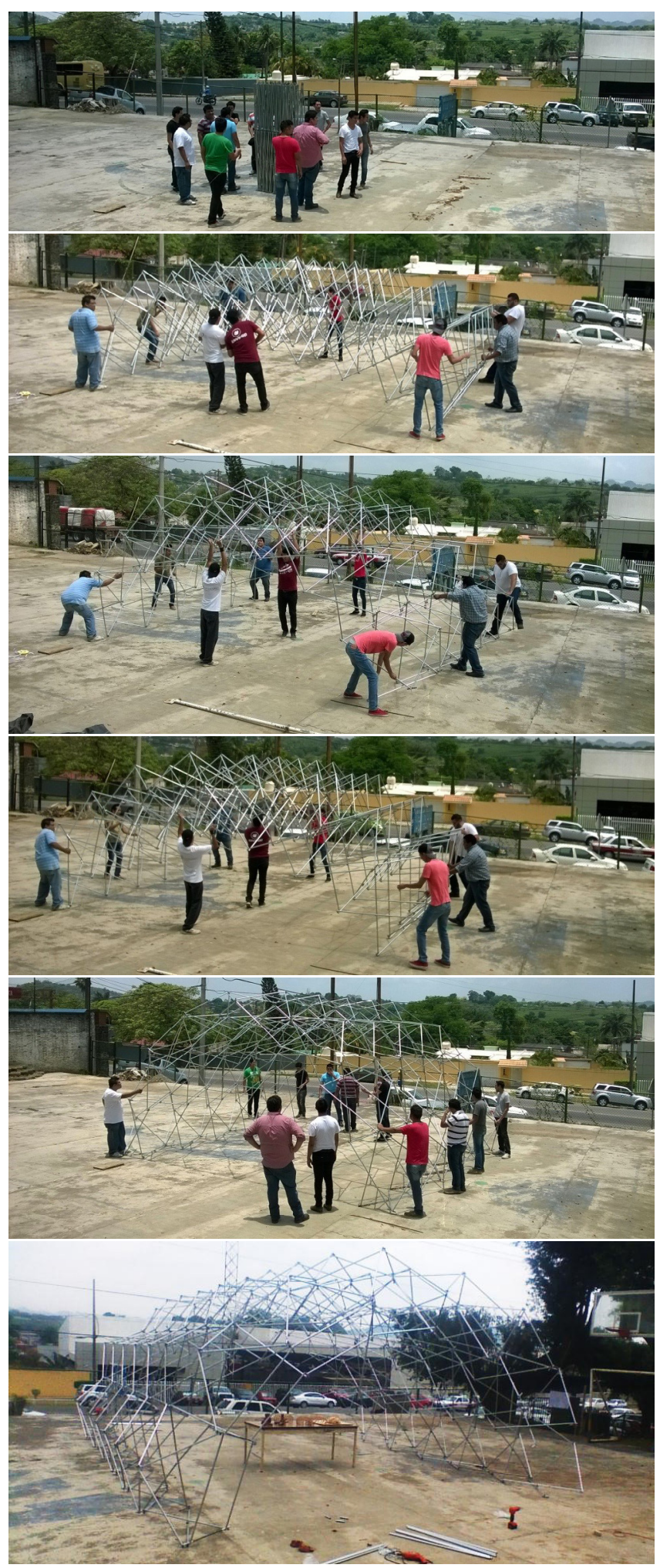

Figuras 17 a la 22. El proceso de alzado ayuda a determinar cómo se comportaría el modelo en la construcción y como serían sus deformaciones en algunas partes de la cubierta, también se comprobó que se puede unificar el sistema transformable, ya que anteriormente está separada por la cubierta plegable de cañón corrido y una cúpula rebajada de costado como dos sistemas independientes, con esta práctica determinamos que pueden estar unidas. Fuente: Morales (6) (10) (15) (26)
En esta etapa se realizó el desplegado ejecutado por medio de un jalón, este desarrolla esfuerzos de sobre tension en las aspas de la base de la estructura, por ello se tendrá que hacer con mucho cuidado el despliegue. Una de las principales características del modelo después del despliegue es que se equilibra por su geometría, para llegar a esto, se contempla que la estructura debe estar correctamente transformada, ya que los arrostramientos o contra venteos, ayudan a la rigidez de la cubierta si está completamente erguida.

Otro factor que se tiene que tomar en cuenta es que el sitio debe de estar nivelado ya que para este modelo no se realizaron los basamentos de nivelación que ayudan a adaptarse la cubierta al terreno. En este ejemplo se nota que en diferentes escalas a partir de 1:4, la cubierta puede llegar a tener diferentes utilidades, aunque este analizada para claros más grandes, ya que por su facilidad constructiva le ayuda a que sea muy fácil de instalarla.

Por último, este modelo ayudo a detectar como se iba a desarrollar el alzado de la estructura en la construcción, y cuales serían las complicaciones de su alzado como se comprobó que las aspas de las bases en especial solo las de los extremos de cada esquina, se deformaban un poco a la hora de conectar la unión a la base del suelo, por ello se optara aumentar un poco el perfil en esas partes, para concluir se demostró que la geometría unificada tanto de la cubierta central semicircular como las cúpulas circulares de los constados se pueden desplegar en una sola estructura sin tener que realizar dos alzados, esto definió que la cubierta es viable para ser ejecutada en un solo paquete. (6) (9) (13) (14) (16)

\section{PROPUESTA FINAL}

La síguete propuesta se realiza gracias a la anterior experimentación constructiva, también los cálculos ayudaron a definir las dimensiones adecuadas para las aspas y a formar accesorios y nodos adecuados para un sistema plegable más adecuado. En esta etapa la simulación de la estructura con WinTess se refleja en la propuesta final, (figura $23,24)$ y su unificación de las aspas de la cubierta central con las cúpulas de los extremos forman una sola estructura que se puede izar en un despliegue y no en dos como en la propuesta geométricas anteriores, el beneficio es que la construcción sea armada en un solo día.

Por último la estructura ya simplifica con una sola geometría, se unifica el sistema con solo dos tipos de nodos y dos tipos de accesorios de conexión; un nodo para arrostramiento y otro para la cubierta plegable, (figura 25, 26) logrando una estética simplificada, también se simplifica los detalles de conexión del nodo con la membrana, haciendo que la regulación de la cubierta sea más simple y pueda darle la rapidez al desplegado de la estructura, en consecuencia esto mejora el rendimiento y costo de la construcción.

En consecuencia se selecciona el material de la membrana textil basándonos en los resultados del cálculo, (figuras 27 a la 30) esta puede ser de cualquier color; blanca, azul, roja, verde, ya que la membrana textil que se utilizará tiene la capacidad de dejar pasar la luz del sol y un poco la sensación térmica, por lo que se eligió un Serge Ferra- 
ri-Fluitop-T2-1002, con una resistencia de $\mathrm{RK}$ (daN/5cm) 420/420, módulo de Elasticidad (t/m) 50/50. El material de los tubos, nodos y accesorios se realizarán con un acero A36, esta estructura medirá $25 \mathrm{~m}$ en sección transversal y $49.5 \mathrm{~m}$ de longitud, esta nueva dimensión es por la mejora de la geometría realizada en los costados de la cubierta plegable, por lo que la propuesta siguiente se diseñan para ser construida y mejorar el sistema de plegado de este proyecto de investigación. (6) (22) (25) (24)
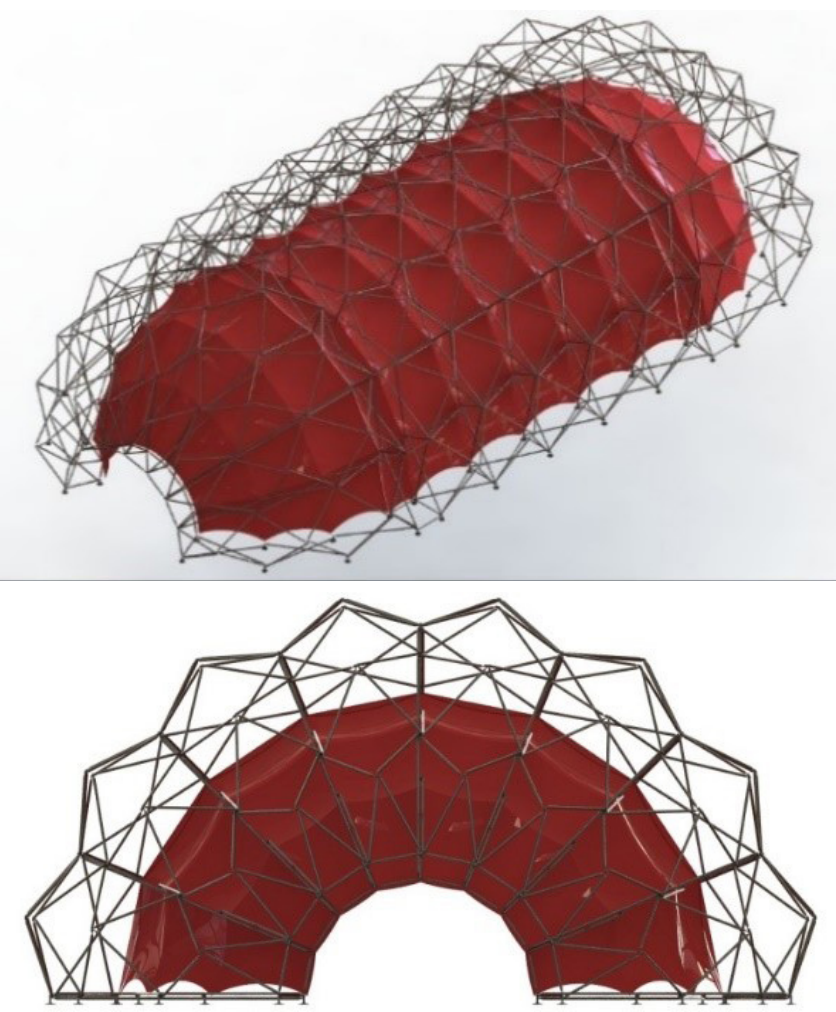

Figuras 23, 24. Forma final del proyecto de investigación de una cubierta plegable tensada. Registrado ante el IMPI (Instituto Mexicano de la Propiedad Industrial) bajo el nombre de "Mejoramiento de un nodo cilíndrico articulado basado en uniones flexibles para una cubierta plegable “. Fuente: Morales, (9) (15)
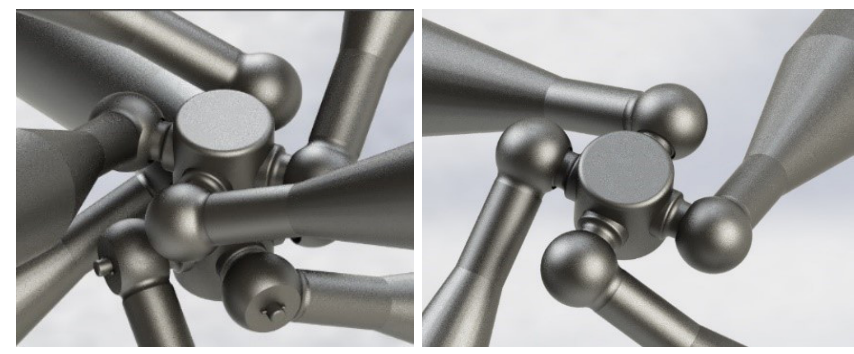

Figuras 25, 26. El detalle estructural se realizó por medio de una simulación en $3 \mathrm{D}$ para verificar los movimientos que podría tener la estructura. Registrado ante IMPI (Instituto Mexicano de la Propiedad Industrial) bajo el nombre de "Mejoramiento de un nodo cilíndrico articulado basado en uniones flexibles para una cubierta plegable”. Fuente: Morales (6) (15)
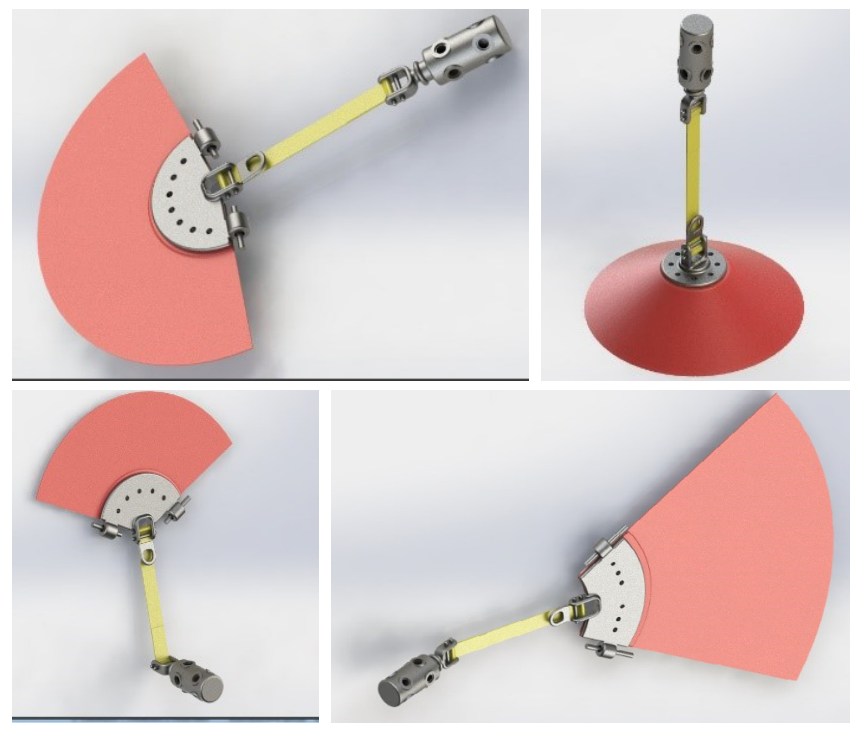

Figuras 27 a la 30. Posteriormente se diseñaron los detalles en $3 \mathrm{D}$ de las uniones de membrana a la estructura. Registrado ante IMPI (Instituto Mexicano de la Propiedad Industrial) bajo el nombre de

"Mejoramiento de un nodo cilíndrico articulado basado en uniones flexibles para una cubierta plegable”. Fuente: Morales (10) (15)

\section{DISCUSION}

Una vez obtenido el diseño final, hacemos una discusión reflexiva sobre la investigación, ya que hay muy pocos trabajos sobre el estudio de los sistemas transformables en la actualidad, para mencionar algunos investigadores; el Dr. Félix Escrig Pallares y el Dr. José Sánchez Sánchez de la Universidad de Sevilla, el Dr. Juan Gerardo Oliva Salinas de la Universidad Nacional Autónoma de México y el Dr. Carlos Hernández de la Universidad de Venezuela, son los que en algún momento desarrollaron referencias constructivas contemporáneas importantes de movilidad mecánica de sistema plegable en las cubiertas, por esta razón, la presente investigación se justificó en la búsqueda de un método que simplificara los métodos de los anteriores referentes en especial de Félix Escrig, ya que su metodología del cual tomamos de guía.

Este estudio ayudo a crear un parteaguas sobre qué tipo de tecnologías y materiales podríamos ocupar, pero con una hipótesis más adecuada para operar los movimientos mecánicos de una estructura plegable, luego se generaron conjeturas respaldadas por los métodos del Dr. Félix Escrig, que ayudaron a realizar un método geométrico más rápido, el cual nos generó un prototipo final de sistema transformable, esto fue un factor muy significativo para una aproximación conceptual, porque sus aportes teórico-práctico que sirvieron para realizar la transición geométrica del proyecto, por lo cual ayudó a construir prototipos de sistemas transformables. Con el desarrollo de esta hipótesis se generó una adecuación geométrica con lo cual se compararon modelos físicos aproximados para su construcción, esto sirvió para validar la etapa siguiente; el análisis estructural.

El estudio del análisis estructural del sistema transformable y su construcción en la arquitectura se basa principalmente en la experimentación. Esto se puede apreciar en la fase de la modelación anterior del prototipo que se realizó para comprobar la movilidad del sistema, la cual se basó en la modelación y verificación por medio de un software (WinTess en 
análisis Estructural), tomando en cuenta las normas (Load and Resistance Factor Desing y Norma de la Construcción del Reglamento del Distrito Federal) y materiales que pudieran mejorar el proceso de la construcción, el cual fue una gran ayuda para realizar el proyecto final; esto da como resultado un sistema estructural configurable a su entorno. Su principio de diseño versó sobre cómo desarrollar un sistema plegable con conexiones articuladas, el cual lograra enlazar mecanismos móviles y resistentes, nos dio la posibilidad de generar aportaciones constructivas para una propuesta final.

Todo esto nos deja diferentes líneas de investigación paralelas para abordar y generar más conocimiento práctico-teórico, un ejemplo de ello es el uso material como el aluminio estructural, aluminio aeroespacial, plástico estructural y acero reforzado con fibra de vidrio, etc. Por lo cual nos daría un bagaje de posibilidades de aplicaciones arquitectónicas, la aplicación de este mismo método se realizaría con otros sistemas plegables u otros sistemas estructurales plegables combinados, guiado por el mapa conceptual re configurado del método de diseño del Dr. Horst Berger, siempre y cuando tengan una base de diseño conceptual basado en referencias, un análisis estructural basado en reglamentos que contemplen algunas condiciones para verificar su viabilidad estructural en un prototipo experimental, y a si se podrá obtener un diseño final de un sistema plegable.

Por último, verificamos el resultado del modelo aproximado, y esto nos deja un parámetro de aplicación constructiva de este tipo de sistemas, los cuales podrán ayudarnos a tener ventajas constructivas en los prototipos de cubiertas plegables, cada detalle constructivo que se generó facilita la manufactura del dispositivo, ya que son relativamente fáciles de montar en sitio, por lo cual se decidió a desarrollar una propuesta final de mejoramiento de nudos, primeramente nuestro modelo no tiene tantas conexiones complejas y su excentricidad con respecto a la geometría se desarrolló con poco desfase, siempre que se diseñen este tipo de estructuras plegables, nos encontraremos con problemas de excentricidades en sus nudos, por lo que se tiene que contemplar para mejorar ese punto de fallo, por ello se simplificó más el nodo para no alberga tantas variantes de conexión y así no alejarse del punto de excéntrico de los miembros estructurales en la unión.

\section{CONCLUSION}

La investigación se basó desde el principio en la metodología de diseño del Dr. Félix Escrig, es punto de referencia en la historia, ya que el desarrollo geometrías estandarizadas que ayudaron a generar sistemas transformables más fáciles de manufacturar en la construcción y rápidos de montar en sitio.

Estas hipótesis de referencias encontradas sirvieron para realizar un sistema plegable, esto fue un factor muy importante para una primeras aproximaciones conceptuales, desarrollados por el método de diseño de Dr. Félix Escrig, donde expuso el desarrollo geométrico y su viabilidad para analizarlas estructuralmente, en consecuencia se propuso un desarrollo geométrico en la tabla 1, que deriva los rasgos principales del método de Escrig, esto ayudo a generar una geometría más rápida para las estructuras plegables, y nos proporcionó una variedad de formas que podemos desarrollar y proponer (figura 31 a la 34 ).
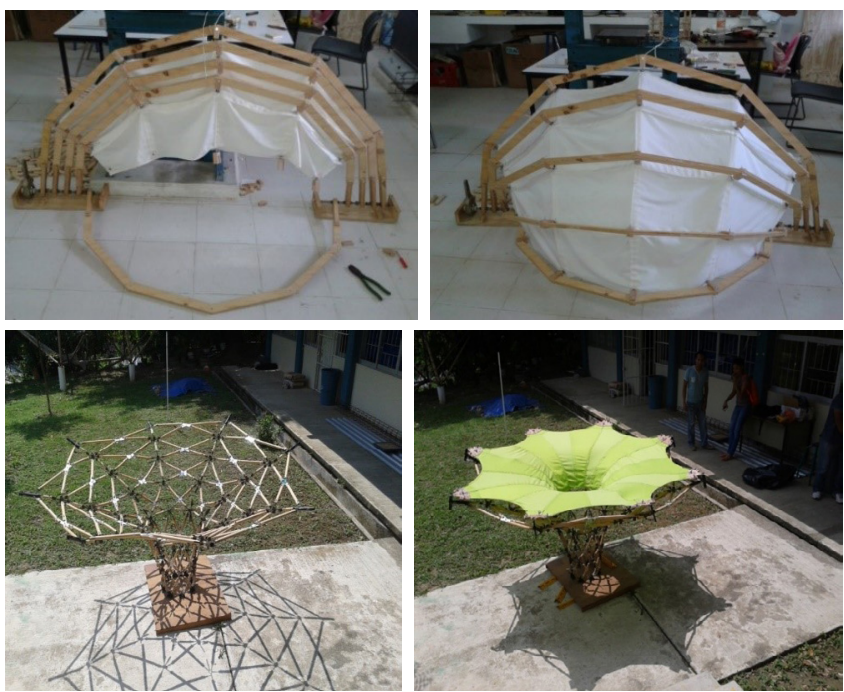

Figura. 31 a la 34. Modelos prototipados para desarrollar geometrías complejas en los sistemas plegables desarrollados en el Laboratorio de Estructuras. Fuente: Morales (8) (9) (10)

Estas suposiciones guiadas por un mapa conceptual desarrollado por el Dr. H. Berger, donde se tiene tres grandes rasgos, Diseño Conceptual, Análisis Estructural y Diseño Final, estas aportaciones teóricas-prácticas se toman como un principio formal para cómo desarrollar un prototipado de las estructuras plegables, generados por parámetros de desarrollo geométrico; esto se compara con propuesta aproximada de conexiones, por lo que a futuro se requiere abordar más sobre el tema para posibles aportaciones extras en la siguiente investigación en el tema de modelos físicos experimentales.

Posteriormente, las ideas expuestas en el diseño conceptual de las hipótesis coadyuvaron a crear un modelo físico y geométrico para construir una estructura plegable, estás solo se implementarán para el desarrollo de un principio de diseño estructural que pueda crear un sistema plegable, dichos modelos generan una premisa teórica constructiva para desarrollar este tipo de sistemas. Todo esto, ofreció un parámetro para desarrollar una propuesta constructiva de dicha estructura plegable, los cuales sirven para verificar y validar nuestra propuesta de adecuación geométrica y extrapolar la información en programas informáticos para realizar con más rapidez el análisis estructural de estos sistemas.

Para el cálculo de la estructura se utilizó la simulación por medio de un software llamado WinTess que realizo un análisis de segundo orden, ya que este tipo de estructuras tiende a ser deformables por sus conexiones flexibles, dado que requieren de mecanismos en las uniones articuladas para materializar su movilidad y puedan retornar a su estado inicial. Finalmente, con los parámetros de combinaciones anteriormente mencionado, se obtiene un planteamiento para proponer y definir aproximaciones de resultados óptimos para el diseño constructivo del prototipo, con el objetivo de demostrar la viabilidad de la propuesta final.

En cuanto a las aplicaciones tecnológicas en la fase de diseño final, se realizó una propuesta aproximada para el prototipo final, esta se deriva en la construcción parcial de modelos a escala real construido con herramientas muy básicas, pero analizado con el rigor científico que sustenta su resistencia 
y equilibrio para armarlo y, con ello, se cotejan aspectos tales como su armado y correcciones de nudos, los alcances de estos modelados ayudaron a adquirir experiencia y conocimiento en la manufactura de los miembros estructurales del modelo.

Esta característica critica ayudó en gran medida a mejorar ciertos criterios de diseño constructivos, el cual se vio reflejado en la construcción final de un modelo que no tuviera tantas excentricidades por medio de uniones sencillas, primeramente, para generar los detalles finales se utilizó un software de diseño industrial y de mecánica llamado Solid Work, estos diseños se adaptaron de acuerdo a las posibilidades de manufactura de la región, ya que existen muchas carencias constructivas y costo del material; a pesar de ello, realizamos varios parámetros y premisas para re configurar las conexiones constructivas finales.

Sin embargo, el logro de crear un modelo final a escala 1:4, dio la pauta de cuáles serían los inconvenientes en estos sistemas, y tratar de subsanar el problema a tiempo para poder fabricar el prototipo final con mucha mayor funcionalidad constructiva, por esta razón los objetivos se vieron cumplidos gracias a que se fabricó un nodo optimo, con las menores fallas de excentricidades para manufacturar y la sencillez de armado para su posterior montaje en la construcción. Con la idea de obtener mejores resultados en la movilidad mecánica y no haya tanto desfase excéntrico en sus conexiones de armado con los miembros de la estructura.
Aunque no todo está concluido en esta investigación, hay puntos que tendrán que estudiarse más a fondo, y así realizar un estudio más detallado, como es el caso de la adaptación de los sistemas bidireccionales o el desarrollo de la geodésica rebajada plegable, las diferentes tipologías encontradas en el diseño de sistemas plegables y su variedad de adaptaciones geométricas que pudieran llegar obtener un sistema plegable (figura. 35 a la 38).

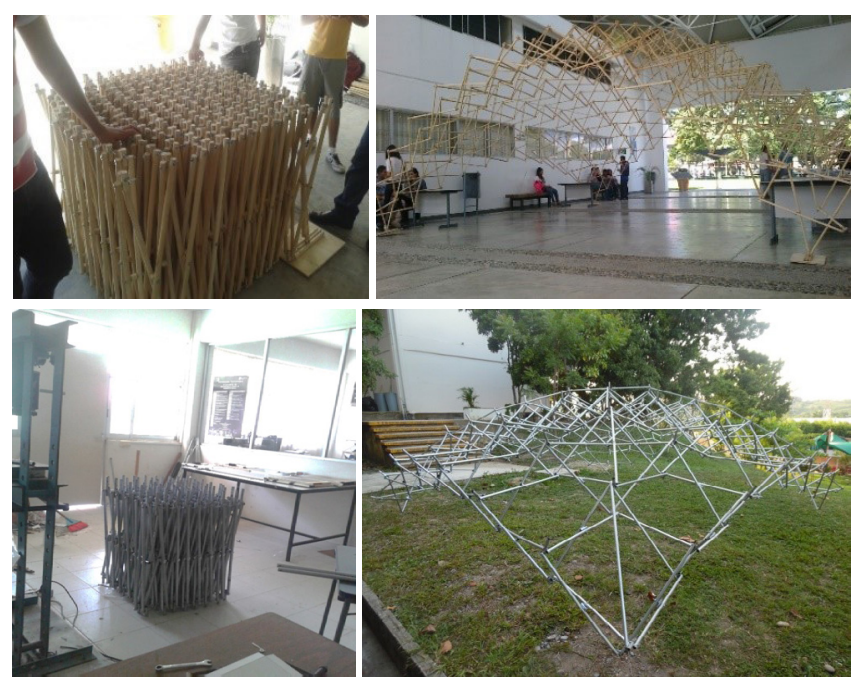

Figura. 35 a la 38. Propuestas para el Ayuntamiento para rescate de espacios públicos desarrollados por vinculación con el Laboratorio de Estructuras. Fuente: Morales (10) (13) (14) (16) (17) (23)

\section{REFERENCIAS}

(1) Morales, C.C (2017). Experiencias y casos de estudio: construcción de una cubierta hiperbólica tensada para espacios tropicales. En Pesantez, G. y Flores, W. (comps.). Arquitectura: experiencias y propuestas para la ciudad (pp. 177-192). Guayaquil: Centro de Investigaciones y Desarrollo de Ecuador.

(2) Sánchez, F. (1992). Estructuras Ligeras para Grandes Luces. 1 ${ }^{\mathrm{a}}$, Ed: Editorial Fundación Emilio Pérez Piñero.

(3) Schock, H. J. (1997). Soft Shells, Desing and technology of Tensil Architecture. 1st Ed: Berlín, Germany: Editorial Birkhäuser Verlag.

(4) Berger, H. (1996). "Light Structures, Structures of light, the art of tensile Architecture.” 1st Edition. Switzerland: Basel.

(5) Engel, H. (2006). Sistema de Estructuras. 4a Ed.; México. Editorial Gustavo Gili, S.A.

(6) Morales, C.C. (2009). Diseño de Sistemas Geométricos Estructurales Flexibles para el Espacio Arquitectónico, Tesis de Doctorado en Arquitectura. Área: Tecnología. Universidad Nacional Autónoma de México, Ciudad de México. México

(7) Fuller, M. (2000): Compresión de las Estructuras en la Arquitectura. 1a Ed.: Editorial interamericana Mcgraw-Hill.

(8) Morales, C. C (2018) Diseño y desarrollo de patrones de la forma de una tenso-estructura. Revista de Arquitectura. Vol. 21, ISSN: 2357-626X Editorial Universidad Católica de Colombia, Colombia. https://doi.org/10.14718/RevArq.2018.20.1.2005

(9) Morales, C. C (2019) Diseño y construcción de un paraguas plegable para espacios arquitectónicos. Revista de Arquitectura. Vol. 22, ISSN: 2357-626X Editorial Universidad Católica de Colombia, Colombia. https://doi.org/10.14718/ RevArq.2019.21.1.1623

(10) Morales, C.C. (2007). Sistemas Flexibles en la Vivienda. Tesis de Maestría en Diseño Arquitectónico y Bioclimatismo. Universidad Cristóbal Colon, Veracruz, México.

(11) Escrig, F. (1988), Estructuras espaciales desplegables curvas, Revista Informes de la Construcción, Vol. 39, ISSN:19983234, Editorial Consejo Superior de Investigaciones Científicas.

(12) Escrig, F. (1984), Estructuras espaciales de barras desplegables, Revista Informes de la Construcción, Vol. 36, ISSN:19983234, Editorial Consejo Superior de Investigaciones Científicas.

(13) Morales, C. C (2016a) Construcción Experimental de un sistema Transformable Tensado Plegable. Revista de Arquitectura, Vol. 18, ISSN: 2357-626X Editorial Universidad Católica de Colombia, Colombia. https://doi.org/10.14718/ RevArq.2016.18.1.9

(14) Morales, C. C (2016b) Development and experimental construction of a transformable roof (first phase). Magazine Asian Academic Research Journal of Multidisciplinary, Vol. 3, ISSN: 2319 - 2801. Editorial Asian Academic Research Associates

(15) Morales, C. C. (2012). Diseño de Cubiertas Ligeras Tensadas. Estancia Post Doctoral, Universidad Politécnica de Catalunya.

(16) Morales Guzmán, C. C., Martínez Cruz, v., Rivera Torre, H. and Flores Gutiérrez, A. (2017). Desarrollo de la forma de una tenso-estructura en el diseño por computadora. Vol. 4. ISSN: 2500-5200, Revista Procesos Urbanos, Editorial Corporación Universitaria del Caribe, Colombia. https://doi.org/10.21892/2422085X.356 
(17) Morales, C. C (2013) Prototipo de Diseño de una Cubierta Retráctil Tensada. Revista de Arquitectura, Vol. 15, ISSN: 2357-626X Editorial Universidad Católica de Colombia, Colombia. https://doi.org/10.14718/RevArq.2013.15.1.11

(18) Escrig, F. (2012). Modular, ligero, transformable: un paseo por la arquitectura ligera móvil. Ed, Editorial, Universidad de Sevilla. Sevilla: Universidad de Sevilla.

(19) Gómez, V. (2007). Tensegridad, Estructuras Tensegrícas en Ciencias y Artes. $1^{\mathrm{a}}$, Ed, Editorial Universidad Catalunya.

(20) McCormac, (2000). Diseño de Estructuras de Acero, Método LRFD, 2 edición, Editorial Alfaomega

(21) Segui W. (2000). Diseño de Estructuras de Acero con LRFD. 2a Ed. México: Editorial Thomson Editores, S.A de C.V.

(22) Manual de diseño de WinTess3. Fuente: http://tecno.upc.edu/wintess/manual

(23) Morales, C. C (2014) Proceso de diseño de sistemas transformables En las cubiertas ligeras. Revista Modulo Arquitectura, Vol. 14, ISSN: 2389-7732. Editorial Universidad de la Costa, Colombia.

(24) Solid Works es un programa que realiza modelados industriales en 3d. Fuente: http://www.solidservicios.com/. Consultado 2009-2018

(25) Morales, C. C (2013). Diseño de Cubiertas Plegables Tensadas en la Arquitectura. Tesis de Máster de Ingeniería para la Arquitectura, Área: Estructuras, Universidad Camilo José Cela, Madrid, España.

(26) Manual de diseño Estructural de CFE (Comisión Federal de Electricidad). http://cgservicios.df.gob.mx/prontuario/ vigente/385.htm. 